\title{
Early diagnosis of congenital toxoplasmosis in newborn infants using IgG subclasses against two Toxoplasma gondii recombinant proteins
}

\author{
Carlos Henryque de Souza e Silva', Gláucia Queiroz de Andrade², \\ José Nélio Januário², Ana Carolina de Aguiar Vasconcelos Carneiro', Mariangela Carneiro1, \\ Daniel Vitor Vasconcelos-Santos ${ }^{2}$, Ricardo Wagner de Almeida Vitor ${ }^{1 /+}$ \\ 1'Departamento de Parasitologia, Instituto de Ciências Biológicas ${ }^{2}$ Faculdade de Medicina, \\ Universidade Federal de Minas Gerais, Av. Antônio Carlos 6627, 31270-901 Belo Horizonte, MG, Brasil
}

\begin{abstract}
The aim of this work was to evaluate the utility of ELISA-based testing of total IgG (IgGt) antibodies and its subclasses (IgG1, IgG2, IgG3 and IgG4) against soluble (STAg) and recombinant (rSAG1 and rMIC3) antigens of Toxoplasma gondii for diagnosing congenital toxoplasmosis. Sera from 217 newborns initially testing positive for specific IgM in filter paper dried blood spots were tested for specific IgM and IgG by ELFA-VIDAS ${ }^{\circledR}$. Congenital toxoplasmosis was confirmed in 175 and ruled out in 42 infants. The validity of the ELISA tests was determined using the persistence of IgG antibodies (ELFA-VIDAS ${ }^{\circledR} \mathrm{kit}$ ) at the end of 12 months, which is considered the reference test for the diagnosis of congenital toxoplasmosis. The frequency of positivity with IgGt against STAg, rSAGI and rMIC3 was found in $97.2 \%, 96.3 \%$ and $80.2 \%$, respectively, of the newborns with confirmed congenital toxoplasmosis. IgG1 reacted with all three antigens, while IgG3 and IgG4 reacted preferentially with rMIC3. Higher mean values of reactivity (sample optical density/cut-off) were found for all subclasses when using rMIC3. All of the antigens showed high sensitivity and low specificity in detecting anti-T. gondii IgGt and IgG1 and low sensitivity and high specificity in detecting IgG3 and IgG4. In conclusion, the combined detection of IgG antibody subclasses against recombinant toxoplasmic antigens may be useful for the early diagnosis of congenital toxoplasmosis.
\end{abstract}

Key words: congenital toxoplasmosis - ELISA - recombinant proteins - IgG subclasses

Toxoplasmosis is a worldwide zoonosis caused by Toxoplasma gondii, an intracellular protozoan of a heteroxenic life-cycle that is capable of infecting a variety of warm-blooded vertebrates. Up to $30 \%$ of the human population may harbour the parasite (Tenter et al. 2000). In immunocompetent hosts, toxoplasmosis may be asymptomatic and induces a long-lasting immune response, which is sufficient to prevent the transplacental transmission of the parasite. However, if primary infection occurs during pregnancy, $T$. gondii can be transmitted to the foetus and cause inflammatory lesions, resulting in potentially severe neurological and ophthalmological damage (Petersen 2007). The early diagnosis of congenital toxoplasmosis (CT) may allow for prompt treatment of these children, possibly leading to more favourable outcomes (Altcheh et al. 2006, Vasconcelos-Santos et al. 2009). Thus, serological tests for the detection of antibodies may be valuable tools for the early diagnosis of such infections (Rorman et al. 2006). The use of recombinant antigens in serological tests has advantages over total antigens because they preferentially react with the IgG antibodies of individuals with acute infections ( $\mathrm{Li}$ et al. 2000, Altcheh et al. 2006).

Financial support: SES/MG, FAPEMIG (CBB-APQ-00129-09), CNPq (301110/2009-3)

RWAV is a CNPq research fellow.

+ Corresponding author: ricardovitor@icb.ufmg.br

Received 22 June 2011

Accepted 1 December 2011
This study evaluated the use of soluble (STAg) and recombinant (rSAG1 and rMIC3) T. gondii antigens in ELISA-based testing for total IgG antibodies (IgGt) and its subclasses in serum samples of newborns with congenital toxoplasmosis.

\section{SUBJECTS, MATERIALS AND METHODS}

Patients and clinical samples - This study was conducted as part of a prospective investigation on the impact of CT in the state of Minas Gerais from 1 November 2006-31 May 2007 with children participating in the State Program for Neonatal Screening (Vasconcelos-Santos et al. 2009). In this program, filter paper-dried blood samples from 146,307 newborns were tested for anti-T. gondii IgM (TOXO IgM Q-preven ${ }^{\circledR}$, Symbiosis, Leme, Brazil) with 217 presenting positive or inconclusive serological results. These newborns were retested for IgG and IgM one month after birth (ELFA-VIDAS ${ }^{\circledR}$, BioMérieux AS, Lyon, France) and were classified into two groups: (i) group I (infected children), which comprised 175 infants who had persistent, specific IgG antibodies (ELFA-VI$\mathrm{DAS}^{\circledR}$ ) at 12 months of life and (ii) group NI (non-infected children), which comprised 42 infants who tested negative for specific $\operatorname{IgG}\left(\right.$ ELFA-VIDAS $\left.^{\circledR}\right)$ at the end of the first year of life. The sera used in the ELISA with recombinant T. gondii antigens were collected from infants at an average age of $55.6 \pm 16.6$ days of life. The study protocol was approved by the local Ethical Committee (Federal University of Minas Gerais, protocol 298/06).

Antigens - STAg of T. gondii was obtained as previously described (Elsaid et al. 2001). Briefly, tachyzoites 
of the RH strain were sonicated using five 30 -sec periods at $40 \mathrm{~Hz}$ and $1 \mathrm{~min}$ intervals. After centrifugation at $4^{\circ} \mathrm{C}(30 \mathrm{~min}$ at $15,000 \mathrm{~g})$, the supernatant was collected and used as STAg. Additionally, two commercially available recombinant $T$. gondii antigens (SAG1 and MIC3, GenWay Biotech, Inc, San Diego, CA) were used in the ELISA for the diagnosis of CT. Recombinant antigens were expressed in Escherichia coli as fusion proteins with glutathione S-transferase (GST).

ELISA - Maxisorb plates (Nunc, Denmark) were sensitised overnight with $100 \mu \mathrm{L}$ of STAg $(10 \mathrm{ug} / \mathrm{mL}), \mathrm{rSAG} 1$ $(2.5 \mathrm{ug} / \mathrm{mL})$ or $\mathrm{rMIC} 3(2.5 \mathrm{ug} / \mathrm{mL})$ and blocked for $1 \mathrm{~h}$ at $37^{\circ} \mathrm{C}$ with $2 \%$ foetal calf serum in phosphate buffered saline, $\mathrm{pH}$ 7.2. Serum samples were diluted to previously determined optimum titres and incubated for $1 \mathrm{~h}$ at $37^{\circ} \mathrm{C}$ (STAg: IgGt - 1:400, IgG1 - 1:100, IgG2, IgG3 and IgG4 - 1:50; rSAG1: IgGt, IgG1, IgG2 and IgG3 - 1:100, IgG4 - 1:50; rMIC3: IgGt, IgG2, IgG3 and IgG4 - 1:50, IgG1 - 1:100). After washing, the plates were incubated with biotin-conjugated murine monoclonal anti-human antibody (anti-IgGt, - -IgG1, -IgG2, -IgG3 or -IgG4) at a previously determined optimum dilution for $1 \mathrm{~h}$ at $37^{\circ} \mathrm{C}$ (STAg: IgGt - 1:20,000, IgG1 - 1:8,000, IgG2, IgG4 - 1:5,000 and IgG3 - 1:4,000; rSAG1: IgGt - 1:20,000, IgG1, IgG2, IgG4 - 1:5,000 and IgG3 - 1:10,000; rMIC3: IgGt, IgG1, IgG4 - 1:5,000, IgG2 and IgG3 - 1:2,500). A solution of streptavidin-peroxidase (SIGMA), diluted 1:4,000, was then added. After $30 \mathrm{~min}$ at $37^{\circ} \mathrm{C}$, the plates were washed and incubated with the chromogen (o-phenylenediamine in $0.1 \mathrm{M}$ citric acid, using hydrogen peroxide as the substrate) for $20 \mathrm{~min}$. The assay was interrupted by the addition of $4 \mathrm{~N}_{2} \mathrm{SO}_{4}$ and the absorbance was read at $492 \mathrm{~nm}$ (reference wavelength at $650 \mathrm{~nm}$ ) using an ELISA reader (BIO-RAD 3550). In the readings obtained with the two recombinant proteins, the absorbance value for each serum was subtracted against readings taken of $2.5 \mu \mathrm{g} / \mathrm{mL}$ GST (SIGMA) which was used to sensitise parallel wells of the same plate. The cut-off value was considered the mean absorbance of eight samples of human sera negative for T. gondii (for each isotype) plus three standard deviations tested on each plate. Each serum sample was assayed in duplicate. The results were expressed as the reactivity index (RI) (average sample optical density/cut-off). The sera with RI values equal to or greater than 1 were considered positive (Altcheh et al. 2006).

Statistical analysis - The validity of the ELISA tests was determined by a comparison with the results of the reference test, IgG ELFA-VIDAS ${ }^{\circledR}$. The persistence of specific IgG antibodies at 12 months of life confirmed congenital toxoplasmosis. The sensitivity and specificity of the tests were determined, as well as the positive and negative likelihood ratios (LR) and respective $95 \%$ confidence intervals. A chi-squared test was used to compare the frequencies between infected and noninfected children $(\mathrm{p}<0.05)$. Differences between serum reactivity for the different antigens were analysed using the McNemar test. The mean RI values for IgGt and subclasses against the different antigens were compared by Student's $t$ test $(\mathrm{p}<0.05)$.

\section{RESULTS}

Initially, IgGt and its subclasses reacting with STAg, rSAG1 and rMIC3 were assessed in the sera of the 217 children positive for IgM. The frequency of positivity for IgGt was higher with STAg (97.2\%) and rSAG1 (96.3\%) compared with rMIC3 (80.2\%) (Fig. 1). Similar results were observed for IgG1, while for IgG2 and IgG3 the frequency of positivity against rMIC3 was higher than that against rSAG1. IgG4 reacted more frequently with rMIC3 $(51.2 \%)$ than with STAg $(33.6 \%)$ or rSAG1 $(17.5 \%)$.

All sera reacted with at least one of the antigens. However, different mean RI values were observed for IgGt and its subclasses (Fig. 2). IgGt was less reactive with rMIC3 than with STAg or rSAG1. For the IgG subclasses, the mean RI values were higher for rMIC3. IgG antibodies to rSAG1 were least reactive. The reactivity of sera obtained from 175 children with confirmed CT (group I) was compared with that of the 42 non-infected (group NI) control children, whose antibodies had been passively acquired from the mother. The ELISA for IgGt showed reactivity in more than $84 \%$ of infected infants, regardless of the antigen used (Table I). IgGt in noninfected infants reacted with less intensity than in infected infants with either the rSAG1 or rMIC3 antigen. For IgG1, the proportion of non-infected infants recognising the three antigens was significantly lower than that of the infected children. For IgG2, IgG3 and IgG4, reactivity was moderate, with significant differences between the proportion of positive ELISAs for infected and non-infected children, especially when using rMIC3. For $\operatorname{IgG} 4$, only the reaction with rMIC3 showed significantly different frequencies between the infected $(62.3 \%)$ and non-infected (4.8\%) newborns (Table I).

Table II shows the sensitivity and specificity values and positive and negative LRs of the ELISA-based testing for IgGt and its subclasses in the sera of the infected and non-infected newborns, considering the persistence of $\operatorname{IgG}\left(\right.$ ELFA-VIDAS $\left.^{\circledR}\right)$ at 12 months of life as the reference (gold standard). Better sensitivity and negative LR results were found for IgGt and IgG1, particularly against STAg or rSAG1. Better specificity and positive LR results were observed for $\operatorname{IgG} 3$ and $\operatorname{IgG} 4$, particularly against rMIC3.

\section{DISCUSSION}

In this study, we analysed the presence of IgGt and its subclasses through an ELISA of serum samples from 217 newborns with suspected CT. The IgG antibodies from these children reacted with STAg and the recombinant proteins SAG1 and MIC3. IgGt sensitivity varied between $86-99 \%$. In contrast, the specificity ranged from $7-35 \%$, depending on the antigen employed. A significant difference was observed when using recombinant antigens when comparing the proportions of the reactive samples of the infected and non-infected newborns. Similar results were observed by Buffolano et al. (2005), who found that over $75 \%$ of the samples from infected children reacted with the recombinant antigens.

Similar to IgGt, the ELISA for IgG1 showed high sensitivity and low specificity, indicating that the test is able to identify infected newborns, but cannot accurately differentiate them from non-infected ones. The 
negative LRs for IgGt and IgG1 were low, indicating that the probability is high that a newborn testing negative is not infected. The high IgG levels (mainly IgG1) in these newborns can be explained by the passive transfer of maternal antibodies during pregnancy, which decrease after birth (Correa et al. 2007, Petersen 2007).

For $\operatorname{IgG} 2$, low sensitivity and specificity were observed using STAg (61\% and 64\%, respectively), while rMIC3 showed a higher sensitivity (95.9\%). This result could explain the significant difference between the proportions of positive results for infected and uninfected children using STAg vs. rMIC3. The IgG3 and IgG4 subclasses revealed higher specificities (despite a low sensitivity) and higher positive LRs on the ELISA when compared with IgGt. These results show that children with a positive ELISA are probably infected, as $\operatorname{IgG} 2$ and $\mathrm{IgG} 3$ to $T$. gondii are synthesised by the newborns and their presence indicates an active response against infection (Cañedo-Solares et al. 2008). As observed for IgG2, a significant difference between the proportion of posi- tive ELISAs for IgG3 in the infected and non-infected newborns was found using STAg or rMIC3. ELISA for IgG4 using rMIC3 also showed a significant difference between the proportion of positive tests for the infected and non-infected infants. Only 5\% of the non-infected newborns reacted with rMIC3, while $62 \%$ of the infected children reacted with this antigen.

Higher mean RI values were observed for all IgG subclasses on the ELISA using rMIC3 when compared with using STAg or rSAG1. Our results show that rMIC3 reacts more frequently with $\operatorname{IgG}$ antibodies in children with CT, despite those non-infected newborns presenting passively acquired maternal antibodies. This finding suggests that anti-MIC3 antibodies may indicate the occurrence of active $T$. gondii replication in infected individuals, as previously described (Beghetto et al. 2005, Buffolano et al. 2005).

In general, all of the sera samples from the children reacted with at least one recombinant antigen, a finding also observed by Buffolano et al. (2005), showing
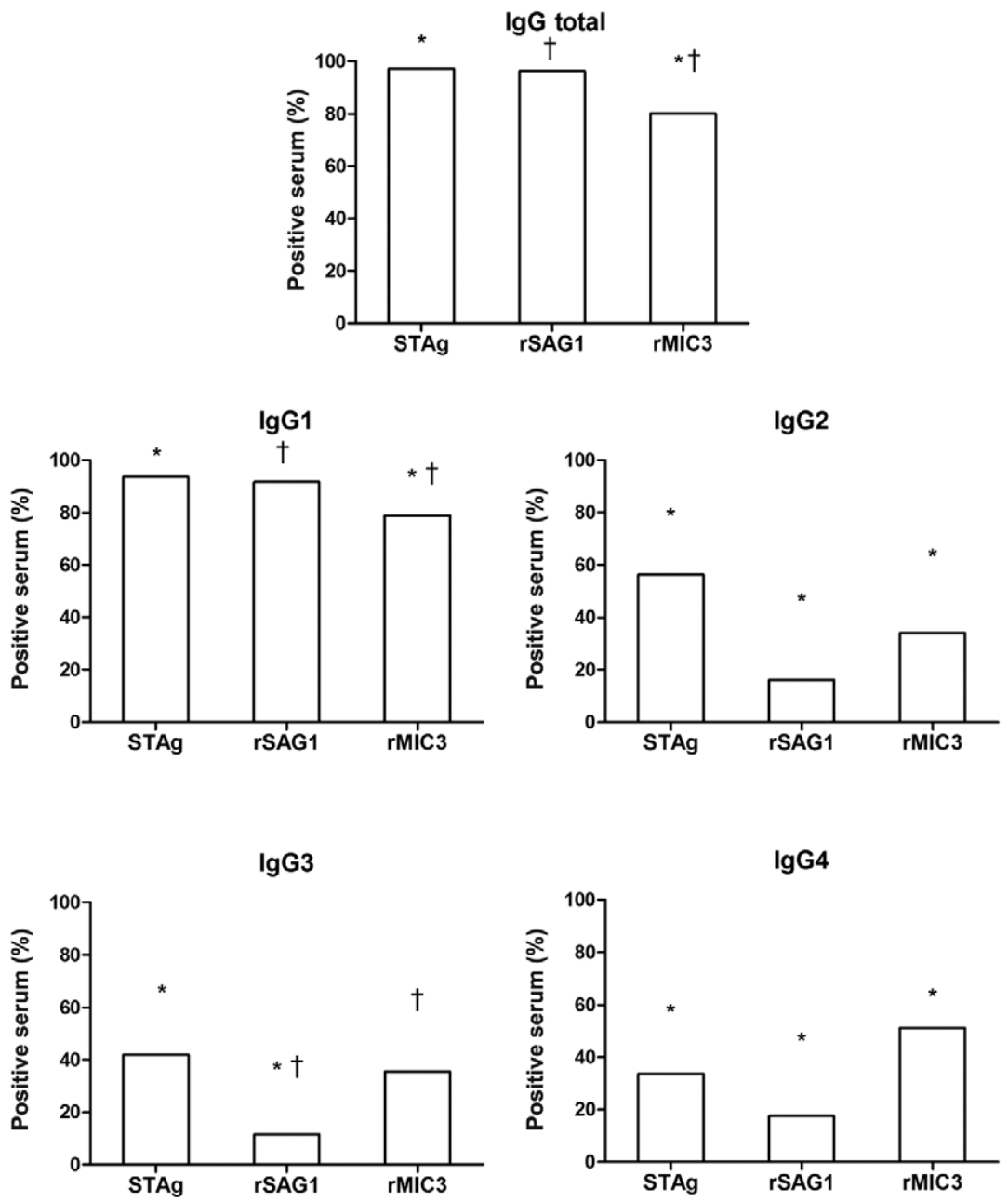

Fig. 1: prevalence of IgG antibody response and its subclasses against soluble (STAg) and recombinant ( rSAG1 and rMIC3) antigens in newborns with suspected congenital toxoplasmosis. The serum samples were considered positive when the values of the index of reactivity (IR) were above 1.0 total $\operatorname{IgG}(\mathrm{IgGt}), \operatorname{IgG1}, \operatorname{IgG} 2, \operatorname{IgG} 3$ and $\operatorname{IgG} 4$. Matching symbols above columns indicate statistical difference $(\mathrm{p}<0.05)$ in the proportion of positive sera using different antigens (McNemar test). 
the immunogenicity of these antigens. Regarding the subclasses, $98 \%$ of the samples tested for IgG1 reacted to at least one of the recombinant antigens, followed by $61 \%$ for $\operatorname{IgG} 4,48 \%$ for $\operatorname{IgG} 2$ and $41 \%$ for IgG3 (data not shown). When testing samples of infected adult patients using an ELISA-STAg, Huskinson et al. (1989) observed that infection with $T$. gondii caused a predominantly IgG1 response. A less intense response was observed for subclasses IgG2 and IgG3, while IgG4 was not recognised. In our study, similar results were also observed when using STAg, rSAG1 and rMIC3. However, we also detected considerable levels of IgG4 reacting with rMIC3.
Our study has some limitations. Newborns were diagnosed with CT by postnatal screening and because the sensitivity of the TOXO IgM Q-preven ${ }^{\circledR}$ and ELFAVIDAS ${ }^{\circledR}$ kits is not very high, the prevalence of infection may have been underestimated. Gilbert et al. (2007) observed the highest sensitivity of postnatal screening for $T$. gondii-specific $\operatorname{IgM}$ and $\operatorname{IgA}$ when tests were performed between one and two weeks after birth. Specificity varied depending on the time of the sampling of newborns and was higher by four weeks after birth. In the present study, newborns were tested one month after birth and this delay may have resulted in false-negative cases that
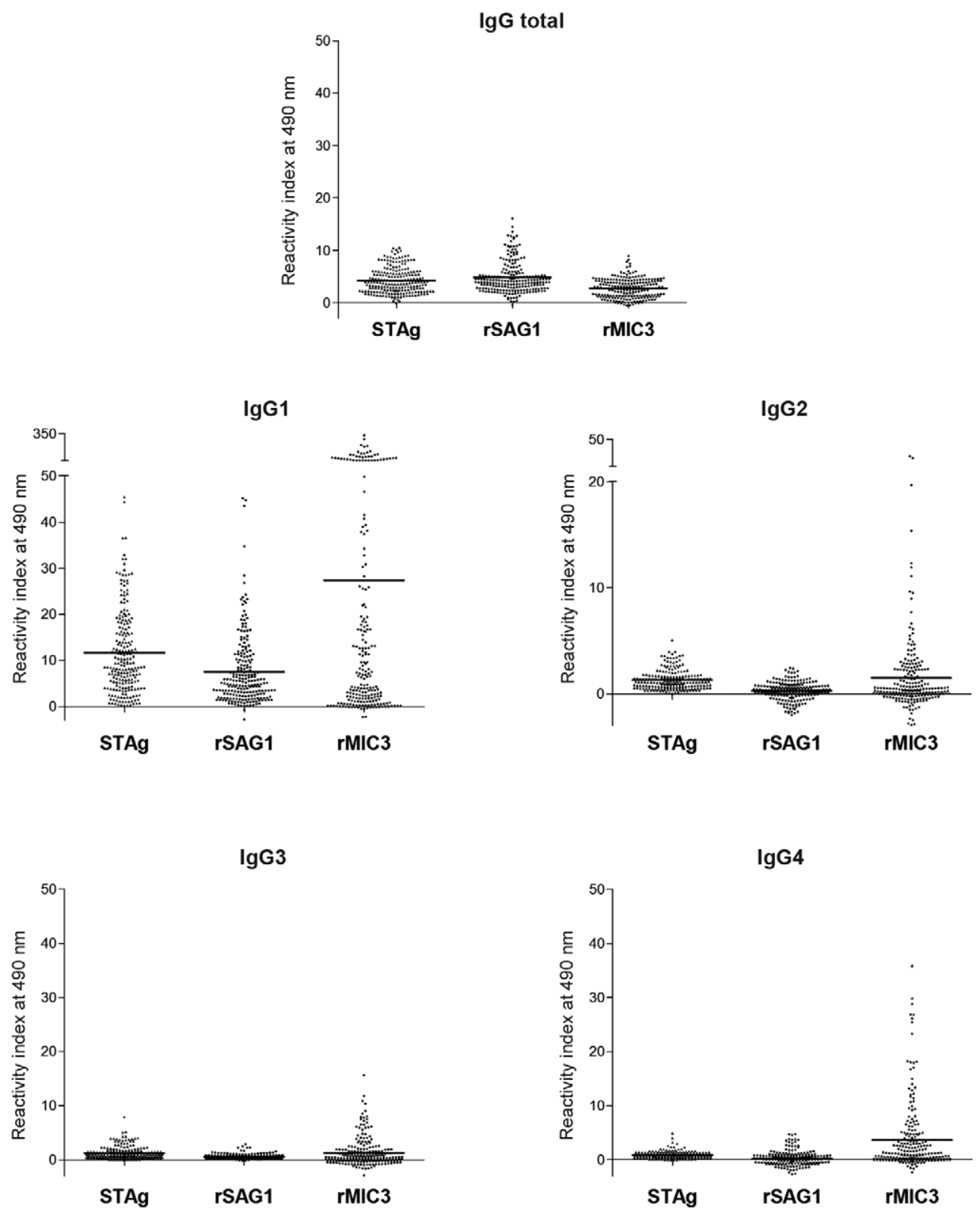

Fig. 2: reactivity index on ELISA of anti-Toxoplasma gondii total $\operatorname{IgG}$ (IgGt), IgG1, IgG2, IgG3 and IgG4, using soluble (STAg) and recombinant (rSAG1 and rMIC3) antigens in children with congenital toxoplasmosis. The continuous horizontal lines correspond to the mean of determinations. Reactivity index: optical density of the serum test/cut-off. 
had been infected in the first trimester of pregnancy and may have ultimately had more severe disease.

In addition to ELISA using recombinant antigens, immunoblot is also being currently evaluated for the postnatal diagnosis of CT. Immunoblot identifies infected newborns with a sensitivity and specificity of $82.4 \%$ and 93\%, respectively (Gross et al. 2000). These results still leave approximately $18 \%$ of congenitally infected newborns without diagnosis confirmation. Difficulties in the standardisation of antigens for immunoblot also hinder its wider application in the serological diagnosis of postnatal CT. However, diagnostic assays based on recombinant antigens for measuring Toxoplasma-specific IgG subclasses show promising results in infants with or without CT born to mothers who have acquired toxoplasmosis during pregnancy (Buffolano et al. 2005).

Our results suggest that the assessment of IgG subclasses using recombinant antigens is a promising complementary tool for CT diagnosis, allowing the identification of a large number of newborns infected with $T$.

\section{TABLE I}

Reactivity of anti-Toxoplasma gondii total $\mathrm{IgG}$ (IgGt) and $\mathrm{IgG}$ subclasses in 217 serum samples: 175 infected children (group I) and 42 non-infected newborns (group NI)

\begin{tabular}{|c|c|c|c|c|}
\hline & \multirow[b]{2}{*}{ Group } & \multicolumn{3}{|c|}{$\begin{array}{l}\text { Reactive samples on ELISA for different antigens } \\
\qquad \mathrm{n}(\%) \mathrm{p}^{a}\end{array}$} \\
\hline & & STAg & rSAG1 & rMIC3 \\
\hline $\mathrm{IgGt}$ & $\begin{array}{c}\text { I } \\
\text { NI }\end{array}$ & $\begin{array}{c}172(98.3) \\
39(92.9) 0.08\end{array}$ & $\begin{array}{c}174(99.4) \\
35(83.3)<0.05\end{array}$ & $\begin{array}{c}148(84.6) \\
26(61.9)<0.05\end{array}$ \\
\hline IgG1 & $\begin{array}{c}\mathrm{I} \\
\mathrm{NI}\end{array}$ & $\begin{array}{c}168(96) \\
35(83.3)<0.05\end{array}$ & $\begin{array}{c}167(95.4) \\
32(76.2)<0.05\end{array}$ & $\begin{array}{c}158(90.3) \\
13(31)<0.05\end{array}$ \\
\hline $\operatorname{IgG} 2$ & $\begin{array}{c}\text { I } \\
\mathrm{NI}\end{array}$ & $\begin{array}{c}107(61.1) \\
15(35.7)<0.05\end{array}$ & $\begin{array}{c}24(13.7) \\
11(26.2) 0.06\end{array}$ & $\begin{array}{c}72(41.1) \\
2(4.8)<0.05\end{array}$ \\
\hline IgG3 & $\begin{array}{c}\text { I } \\
\mathrm{NI}\end{array}$ & $\begin{array}{c}85(48.6) \\
6(14.3)<0.05\end{array}$ & $\begin{array}{c}22(12.6) \\
3(7.1) 0.42\end{array}$ & $\begin{aligned} 77(44) \\
0(0)<0.05\end{aligned}$ \\
\hline IgG4 & $\begin{array}{c}\mathrm{I} \\
\mathrm{NI}\end{array}$ & $\begin{array}{c}64(36.6) \\
9(21.4) 0.07\end{array}$ & $\begin{array}{c}28(16) \\
10(23.8) 0.25\end{array}$ & $\begin{array}{c}109(62.3) \\
2(4.8)<0.05\end{array}$ \\
\hline
\end{tabular}

$a$ : $\mathrm{p}$ value by $\chi^{2}$ comparing infected and non-infected children; rSAG1 and rMIC3: recombinant antigen; STAg: soluble antigen.

TABLE II

Sensitivity, specificity and likelihood ratio of ELISA testing for anti-Toxoplasma gondii IgG antibodies and their subclasses using recombinant proteins in 217 newborn infants

\begin{tabular}{|c|c|c|c|c|c|}
\hline & & $\begin{array}{l}\text { Sensibility } \\
(95 \% \mathrm{CI})\end{array}$ & $\begin{array}{c}\text { Especificity } \\
(95 \% \mathrm{CI})\end{array}$ & $\begin{array}{c}\text { LR } \\
\text { positive }\end{array}$ & $\begin{array}{c}\text { LR } \\
\text { negative }\end{array}$ \\
\hline \multirow[t]{5}{*}{ STAg } & IgG total & 98.8 (95.9-99.7) & $7.1(2.4-19.0)$ & 1.065 & 0.160 \\
\hline & $\operatorname{IgG1}$ & $97.1(93.4-98.7)$ & $16.6(8.3-30.6)$ & 1.165 & 0.173 \\
\hline & $\mathrm{IgG} 2$ & $61.4(54.1-68.4)$ & $64.2(49.2-77.0)$ & 1.722 & 0.599 \\
\hline & IgG3 & $46.5(39.3-53.9)$ & $85.7(72.1-93.3)$ & 3.259 & 0.623 \\
\hline & IgG4 & $36.4(29.6-43.81)$ & $78.5(64.0-88.3)$ & 1.699 & 0.802 \\
\hline \multirow[t]{5}{*}{ rSAG1 } & IgG total & 99.4 (96.3-99.9) & $16.6(8.3-30.6)$ & 1.193 & 0.034 \\
\hline & $\operatorname{IgG1}$ & $95.4(91.2-97.7)$ & $23.8(13.5-38.5)$ & 1.253 & 0.192 \\
\hline & $\operatorname{IgG} 2$ & $13.7(9.4-19.6)$ & $73.1(58.9-84.7)$ & 0.523 & 1.169 \\
\hline & IgG3 & $12.5(8.4-18.3)$ & $92.8(81.0-97.5)$ & 1.760 & 0.941 \\
\hline & IgG4 & $16.5(11.8-22.8)$ & $76.1(61.5-86.5)$ & 0.696 & 1.095 \\
\hline \multirow[t]{5}{*}{ rMIC3 } & IgG total & $86(80.0-90.4)$ & $34.8(22.4-49.8)$ & 1.321 & 0.400 \\
\hline & IgG1 & $90.2(85.0-93.8)$ & $69.0(54.0-80.9)$ & 2.917 & 0.140 \\
\hline & $\mathrm{IgG} 2$ & $95.9(88.7-98.6)$ & $27.2(20.6-35.1)$ & 1.319 & 0.140 \\
\hline & IgG3 & $44.0(36.8-51.4)$ & $100(91.6-100)$ & ND & 0.560 \\
\hline & IgG4 & $62.2(54.9-69.1)$ & $95.2(84.2-98.7)$ & 13.080 & 0.396 \\
\hline
\end{tabular}

CI: confidence interval; ND: not determined; LR: likelihood ratio; rSAG1 and rMIC3: recombinant antigen; STAg: soluble antigen. 
gondii. Reactivity to the rMIC3 antigen is particularly useful to differentiate infected from non-infected infants. The possibility of standardisation and automation of ELISA with recombinant antigens of $T$. gondii is expected to make this technique feasible for widespread use in the serological diagnosis of postnatal CT.

\section{ACKNOWLEDGEMENTS}

To Rosalida Estevan Nazar Lopes, for her technical assistance.

\section{REFERENCES}

Altcheh J, Diaz NS, Pepe CM, Martin V, Nigro M, Freilij H, Angel SO 2006. Kinetic analysis of the humoral immune response against 3 Toxoplasma gondii-recombinant proteins in infants with suspected congenital toxoplasmosis. Diagn Microbiol Infect Dis 56: 161-165.

Beghetto E, Nielsen HV, Del Porto P, Buffolano W, Guglietta S, Felici F, Petersen E, Gargano N 2005. A combination of antigenic regions of Toxoplasma gondii microneme proteins induces protective immunity against oral infection with parasite cysts. $J$ Infect Dis 191: 637-645.

Buffolano W, Beghetto E, Del Pezzo M, Spadoni A, Di Cristina M, Petersen E, Gargano N 2005. Use of recombinant antigens for early postnatal diagnosis of congenital toxoplasmosis. J Clin Microbiol 43: 5916-5924.

Cañedo-Solares I, Galván-Ramires ML, Luna-Pastén H, Pérez LRR, Ortiz-Alegría LB, Rico-Torres CP, Vela-Amieva M, Pérez-Andrade M, Figueroa-Damián R, Correa D 2008. Congenital toxoplasmosis specific IgG subclasses in mother/newborn pairs. $P e$ diatr Infect Dis $J$ 27: 457-475.

Correa D, Cañedo-Solares I, Ortiz-Alegría LB, Caballero-Ortega H, Rico-Torres CP 2007. Congenital and acquired toxoplasmosis: diversity and role of antibodies in different compartments of the host. Parasite Immunol 29: 651-660.
Elsaid MMA, Martins MS, Frézard F, Braga EM, Vitor RWA 2001. Vertical toxoplasmosis in a murine model. Protection after immunization with antigens of Toxoplasma gondii incorporated into liposomes. Mem Inst Oswaldo Cruz 96: 99-104.

Gilbert ER, Thalib L, Tan HK, Paul M, Wallon M, Petersen E 2007. Screening for congenital toxoplasmosis: accuracy of immunoglobulin $\mathrm{M}$ and immunoglobulin A tests after birth. $\mathrm{J} \mathrm{Med}$ Screen 14: 8-13.

Gross U, Lüder CGK, Hendgen V, Heeg C, Sauer I, Weidner A, Krczal D, Enders G 2000. Comparative immunoglobulin G antibody profiles between mother and child (CGMC test) for early diagnosis of congenital toxoplasmosis. J Clin Microbiol 38: 3619-3622.

Huskinson J, Stepick-Biek PN, Araujo FG, Thulliez P, Suzuki Y, Remington JS 1989. Toxoplasma antigens recognized by immunoglobulin g subclasses during acute and chronic infection. J Clin Microbiol 27: 2031-2038.

Li S, Galvan G, Araujo FG, Suzuki Y, Remington JS, Parmley S 2000. Serodiagnosis of recently acquired Toxoplasma gondii infection using an enzyme-linked immunosorbent assay with a combination of recombinant antigens. Clin Diagn Lab Immunol 7: 781-787.

Petersen E 2007. Toxoplasmosis. Semin Fetal Neonatal Med 12: 214223.

Rorman E, Stein C, Rilkis I, Ben-David H 2006. Congenital toxoplasmosis - prenatal aspects of Toxoplasma gondii infection. Reprod Toxicol 21: 458-472.

Tenter AM, Heckeroth AR, Weiss LM 2000. Toxoplasma gondii: from animals to humans. Int J Parasitol 30: 1217-1258.

Vasconcelos-Santos DV, Azevedo DOM, Campos WR, Oréfice F, Queiroz-Andrade GM, Carellos EVM, Romanelli RMC, Januário JN, Resende LM, Martins-Filho OA, Carneiro ACAV, Vitor RWA, Caiaffa WT 2009. Congenital toxoplasmosis in southeastern Brazil: results of early ophthalmologic examination of a large cohort of neonates. Ophthalmology 116: 2199-2206. 